\title{
COMMENTARY
}

\section{Not sleeping well can damage your heart}

\author{
Masayuki Yoshida \\ Hypertension Research (2010) 33, 535-536; doi:10.1038/hr.2010.53; published online 7 May 2010
}

$\mathrm{O}$ bstructive sleep apnea (OSA) is characterized by recurrent obstruction of the pharyngeal airway during sleep, and results in episodes of intermittent hypoxia. Regardless of the presence of daytime symptoms, OSA is an independent risk factor for cardiovascular disease and increased mortality. ${ }^{1} \mathrm{~A}$ number of studies have shown that development of OSA correlates with increasing weight. The prevalence of OSA among obese patients has been reported, and substantial improvement was achieved by weight reduction. ${ }^{2}$ Regarding the pathophysiology of OSA, an anatomically small pharyngeal airway is a prerequisite factor leading to OSA. This smaller airway size always worsens with increasing body fat and obesity. ${ }^{3}$ When luminal size is narrower, it makes the airway vulnerable to collapse. An individual with OSA is able to compensate for this modified anatomy by increasing the activity of the upper airway muscles during the time that he or she is awake. However, with the onset of sleep, this compensation is lost and airway obstruction occurs. Even short periods of apnea can induce a rise in $\mathrm{PaCO}_{2}$, a fall in $\mathrm{PaO}_{2}$, and increased ventilatory effort against an occluded airway. Therefore, patients with OSA experience repetitive episodes of hypoxia during transient cessation of breathing that promote inflammation and oxidative stress. In addition to direct fat deposits within the airway walls, the upper airway size may be smaller in obese patients because of a reduced lung volume. ${ }^{3}$

A growing body of epidemiological evidence supports an association between OSA and the risk for obesity, insulin resistance and diabetes. ${ }^{4}$ OSA results in decreased insulin sensitivity at peripheral receptor sites, which

Professor M Yoshida is at the Tokyo Medical and Dental University, Life Science and Bioethics Research Center, 1-5-45 Yushima D9, Bunkyoku, Tokyo 113-8510, Japan.

E-mail: masavasc@tmd.ac.jp can eventually lead to exhaustion of pancreatic $\beta$-cell islets after longer periods of OSA. ${ }^{5}$ By contrast, treatment of OSA with continuous positive airway pressure has been shown to improve insulin sensitivity after 3 months. ${ }^{6}$ A previous study comparing lean and obese leptin-deficient mice subjected to intermittent hypoxia showed that obese mice, but not lean mice, exposed to intermittent hypoxia developed a time-dependent increase in fasting serum insulin levels and a worsening of glucose tolerance, indicating insulin resistance. ${ }^{7}$ Nonetheless, the molecular mechanisms linking OSA to insulin resistance remain unclear. Although OSA has been shown to be an independent risk factor for hypertension in population-based cohort studies, the efficacy of continuous positive airway pressure therapy on blood pressure provides somewhat conflicting results.

As mentioned above, OSA and obesity are often comorbid factors that influence metabolic disorders. Therefore, it is sometimes difficult to precisely dissect the effect of OSA from that of obesity. As the majority of obese subjects experienced unrecognized OSA, the pathological importance of OSA may be underestimated. A recent study suggested that the expression level of nuclear factor $-k \mathrm{~B}$ and nitrotyrosine was higher in healthy overweight and obese subjects than in their normal-weight counterparts. ${ }^{8}$ However, the presence of unrecognized OSA may influence inflammatory reactions in these subjects. Thus, it may be useful to exclude comorbid OSA to directly link inflammation and obesity. A recent study showed that central obesity without OSA was not correlated with endothelial dysfunction, inflammation and increased oxidative stress. ${ }^{9,10}$ There is a strong correlation between the extent of endothelial dysfunction and the severity of OSA. This is supported by evidence that OSA itself, but not obesity, induces vascular endothelial dysfunction and inflammation and also elevates oxidative stress (see Figure 1).

People suffering from OSA have been shown to develop more severe cardiovascular disorders, such as hypertension and left ventricular hypertrophy. ${ }^{2}$ Although co-founding variables made it difficult to directly link OSA and left ventricular hypertrophy, a close relationship between these two pathological conditions could dramatically and negatively impact patients' prognoses; therefore, molecular mechanisms that could establish a causal relationship between OSA and left ventricular hypertrophy should be more extensively studied. In this issue of Hypertension Research, Inamoto et al. ${ }^{10}$ examined the effects of statin therapy on intermittent hypoxia-induced left ventricular remodeling in mice, a known model for OSA. They reported that intermittent hypoxia-induced hypertrophy of the cardiomyocytes led to perivascular fibrosis and histological degeneration without affecting systemic blood pressure or plasma cholesterol. They found that this treatment led to an upregulation of tumor necrosis factor- $\alpha$ and transforming growth factor $-\beta$ and to an increase in TdTmediated dNTP nick end labeling-positive myocardial cells after hypoxia. Interestingly, administration of pitavastatin significantly normalized hypertrophic change as well as other histological changes in mice exposed to intermittent hypoxia. They also documented a reduction in tumor necrosis factor- $\alpha$, transforming growth factor- $\beta$ and a number of TdT-mediated dNTP nick end labeling-positive pitavastatin-treated myocardial cells. As the authors discussed, active inflammation induced by intermittent hypoxia may have a role in the development of left ventricular remodeling. Numerous studies, including ours, ${ }^{11,12}$ point to an anti-inflammatory role of statins in addition to their lipid-lowering 


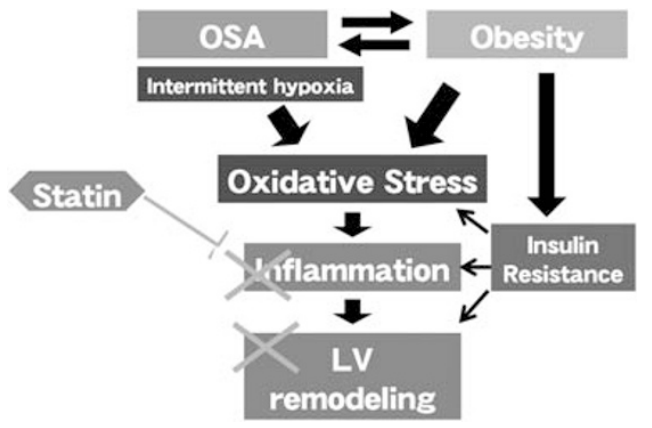

Figure 1 Potential mechanisms of how OSA and obesity lead to left ventricular remodeling. Statin may stabilize inflammation and cardiomyocyte remodeling via a lipid-independent pathway.

effects. Therefore, the anti-inflammatory potential of statins may be beneficial in treating left ventricular hypertrophy. In this context, pharmacological agents such as angiotensin II receptor type 1 blockers may also function to reduce OSA-associated pathological conditions. ${ }^{13}$

It is important to examine how the different temporal patterns of oxidative stress (intermittent vs. constant) affect downstream inflammatory pathways. In addition to the dramatic lipid-lowering effect of pitavastatin, this drug also improves various pathological conditions comorbid to OSA. Therefore, it may be difficult to conclude that the observed beneficial effects of statin therapy resulted from its effect on OSA. More work is required on this topic in the field, as additional information would significantly increase the scientific value of this observation.

1 Formiguera X, Canton A. Obesity: epidemiology and clinical aspects. Best Pract Res Clin Gastroenterol 2004; 18: 1125-1146.

2 Lattimore JD, Celermajer DS, Wilcox I. Obstructive sleep apnea and cardiovascular disease. J Am Coll Cardiol 2003; 41: 1429-1437.

3 Malhotra A, White DP. Obstructive sleep apnoea. Lancet 2002; 360: 237-245.

4 VanHelder T, Symons JD, Radomski MW. Effects of sleep deprivation and exercise on glucose tolerance. Aviat Space Environ Med 1993; 64: 487-492.

5 Vgontzas AN, Papanicolaou DA, Bixler EO, Hopper K, Lotsikas A, Lin HM, Kales A, Chrousos GP. Sleep apnea and daytime sleepiness and fatigue: relation to visceral obesity, insulin resistance, and hypercytokinemia. J Clin Endocrinol Metab 2000; 85: 1151-1158.

6 Harsch IA, Schahin SP, Bruckner K, Radespiel-Troger M, Fuchs FS, Hahn EG, Konturek PC, Lohmann T,
Ficker $\mathrm{JH}$. The effect of continuous positive airway pressure treatment on insulin sensitivity in patients with obstructive sleep apnoea syndrome and type 2 diabetes. Respiration 2004; 71: 252-259.

7 Li J, Grigoryev DN, Ye SQ, Thorne L, Schwartz AR, Smith PL, O'Donnell CP, Polotsky VY. Chronic intermittent hypoxia upregulates genes of lipid biosynthesis in obese mice. J Appl Physiol 2005; 99: 1643-1648.

8 Silver AE, Beske SD, Christou DD, Donato AJ, Moreau KL, Eskurza I, Gates PE, Seals DR. Overweight and obese humans demonstrate increased vascular endothelial NAD(P)H oxidase-p47(phox) expression and evidence of endothelial oxidative stress. Circulation 2007: 115: 627-637.

9 Jelic S, Padeletti M, Kawut SM, Higgins C, Canfield SM, Onat D, Colombo PC, Basner RC, Factor P, LeJemtel TH. Inflammation, oxidative stress, and repair capacity of the vascular endothelium in obstructive sleep apnea. Circulation 2008; 117: 2270-2278.

10 Inamoto S, Yoshioka T, Yamashita C, Miyamura M, Mori T, Ukimura A, Matsumoto C, Matsumura $Y$, Kitaura $Y$, Hayashi T. Pitavastatin reduces oxidative stress attenuates intermittent hypoxia-induced left ventricular remodeling in lean mice. Hypertens Res 2010; 33: 579-586.

11 Laufs U, Endres M, Custodis F, Gertz K, Nickenig G, Liao JK, Bohm M. Suppression of endothelial nitric oxide production after withdrawal of statin treatment is mediated by negative feedback regulation of rho GTPas e gene transcription. Circulation 2000; 102 : 3104-3110.

12 Yoshida M, Sawada T, Ishii H, Gerszten RE, Rosenzweig A, Gimbrone Jr MA, Yasukochi Y, Numano F. Hmg-CoA reductase inhibitor modulates monocyte-endothelial cell interaction under physiological flow conditions in vitro: involvement of Rho GTPase-dependent mechanism. Arterioscler Thromb Vasc Biol 2001; 21: 1165-1171.

13 Yagi S, Aihara K, Ikeda Y, Sumitomo Y, Yoshida S, Ise T, Iwase T, Ishikawa K, Azuma H, Akaike M, Matsumoto T. Pitavastatin, an HMG-CoA reductase inhibitor, exerts eNOS-independent protective actions against angiotensin II induced cardiovascular remodeling and renal insufficiency. Circ Res 2008; 102: 68-76. 